\title{
Design For Manufacture And Assembly Analysis of the Tray for Vertical Lift Modules
}

\author{
Constantin Torcătoru ${ }^{1}$, Dan Săvescu ${ }^{2}$, Narcisa Valter ${ }^{3}$ \\ ${ }^{1,2}$ Product Design, Mechatronics and Environment Department, Transilvania University of \\ Braşov, Romania \\ ${ }^{3}$ Department of Engineering Graphics and Industrial Design, University Politehnica of \\ Bucharest, Romania \\ E-mail: constantin.torcatoru@unitbv.ro
}

\begin{abstract}
This paper addresses the analysis of the design, manufacture, and assembly (DFMA) of the tray for a vertical lift module (Tray VLM), being a subassembly of this automatic VLM storage system. The method used for DFMA analysis is the Boothroyd Dewhurst method, using their software. Based on the recommendations of the DFMA software, following the study on the current Tray VLM product, several components were redesigned using the Design for Manufacture (DFM) module and certain stages for assembly were optimized, using the design for Assembly module (DFA). The result was reduced assembly cost, reduced assembly process time, and increased assembly design efficiency, which were successfully validated by DFMA software by maximizing the DFA index.
\end{abstract}

Keywords: Tray, manufacturing, DFA, DFM, assembly, DFMA, VLM

\section{Introduction}

In today's economic environment, companies are forced to use the best equipment and technology available to manufacture high-quality products at low prices and in a short time. To minimize production costs and product development time, companies have moved from "Traditional Engineering" to "Concurrent Engineering" [1].

Traditional engineering had to deal with the problems that arose because production engineers were not part of the design process.

Simultaneous engineering is a systematic approach to integrated product development, in which work processes overlap and interdisciplinary teams of designers and engineers work in parallel to minimize development time and time to market.

Today, companies need to design and develop products in a fast, accurate, and appropriate way to understand the cost of the product from the design stage.

The DFMA concept aims to bring together all the engineering departments of a manufacturer so that there is a systematic approach to product development.

DFMA has helped manufacturers create products with improved quality, lower costs, and shorter design cycles. DFMA can be applied to a wide range of industries, including automotive, defense, medicine, telecommunications, virtually anywhere there is the development of a new product.

This paper addresses the DFMA method for redesigning the VLM Tray assembly:

- Analysis of the existing VLM Tray assembly, using DFA; 
- Identifying the possibilities for improvement through DFM;

- Modifications to VLM Tray components. to reduce the time and cost of manufacturing and assembly;

- Validation of optimizations through DFA and DFMA.

\section{Methodology}

\subsection{Design for Manufacture and Assembly}

Design for lightweight manufacturing has been considered important for many years, but it was not until the 1970's that the concept of design for manufacturing and assembly began with the research of automatic assembly.

The term "design for manufacturing" (DFM) means design to make manufacturing easier and low cost of all parts that will form the product after assembly and "design for assembly" (DFA) that emphasizes product design for ease of assembly and low cost, thus the "design for manufacturing and assembly" (DFMA) being a merger between DFA and DFM. From a technical point of view, DFMA is defined as a systematic design procedure to analyze and quantify product design [2].

The DFMA concept focuses on three approaches [3]:

- Reducing the number of components of an assembly;

- Redesigning simpler, modular components, easier to process and control, and even cheaper;

- Optimization of production and control technologies;

Any new product development cycle begins with the concepts that appear due to market competition, customer requirements, new manufacturing technologies, etc.

New Product Development (NDP) is a process of continuous innovation in any product enterprise. Continuous updates of creative design are the main priority for manufacturing companies that want to remain competitive in the market at the pace of today's accelerated development.

An effective approach for NPD is to divide into several subassemblies and parts, which can be designed and manufactured in parallel, for the main assembly of the product [4].

As shown in Figure 2.1, it's essential to make the right choices and decisions when designing parts and assemblies from the earliest stage of the NDP, as the cost of change increases dramatically and freedom of choice decreases over time, throughout the manufacturing process, from the design stage) at manufacture (testing).

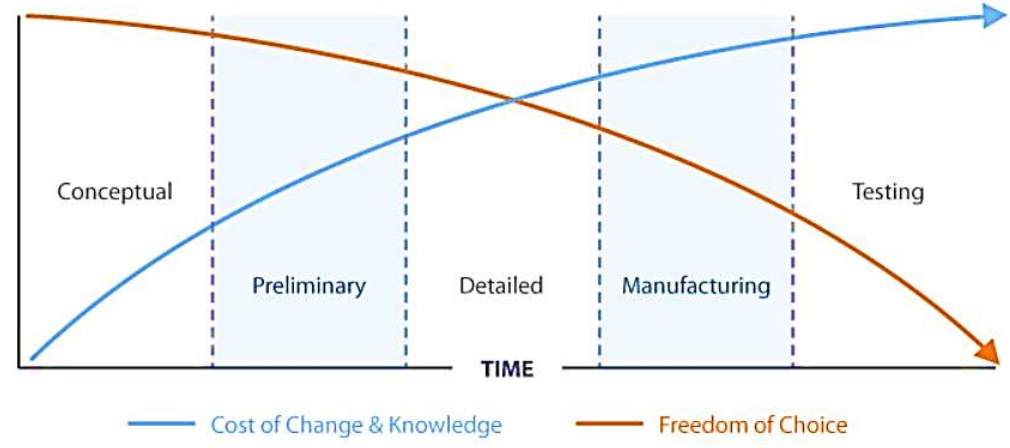

Figure 2.1 New Product Design - Freedom of Choice vs. Cost of Change

Thus, any modification of the project during production results in loss of time, financial and effort. To avoid such cases, special attention must be taken into account for assembly and manufacture, from the design stage, because nowadays it is widely accepted that over $70 \%$ of the costs of the final product are determined during design [2]. 


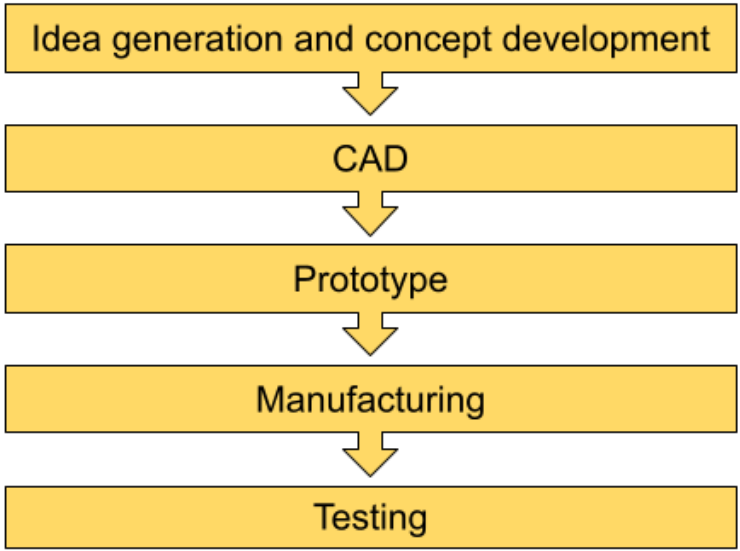

a. Without DFMA;

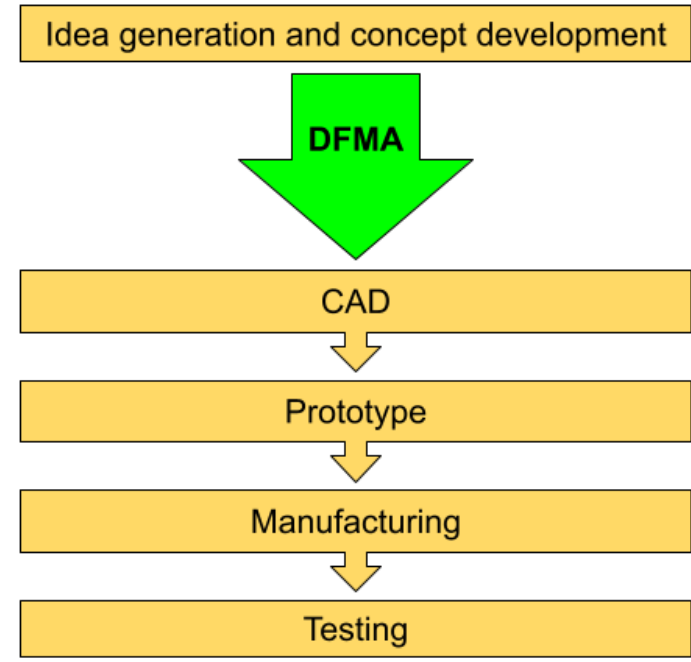

b. With DFMA;

Figure 2.2 Design stages

Figure 2.2 shows where the concept of DFMA analysis plays an important role during product design. The introduction of DFMA in the early design phase helps to find and solve manufacturing and assembly problems, eliminating waste of time, money, and extra effort.

\subsection{DFMA vs. conventional Design Process}

Studies have shown that an increase in the time spent during the concept of developing a product using the DFMA method, can considerably shorten the time to launch the product on the market.

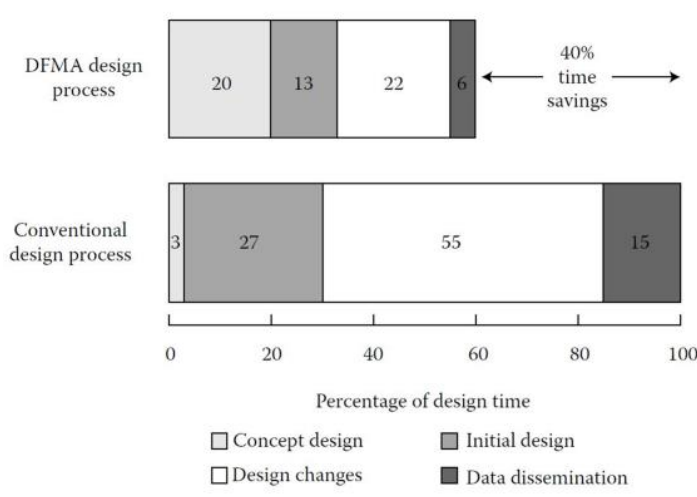

a. DFMA shortens the design process

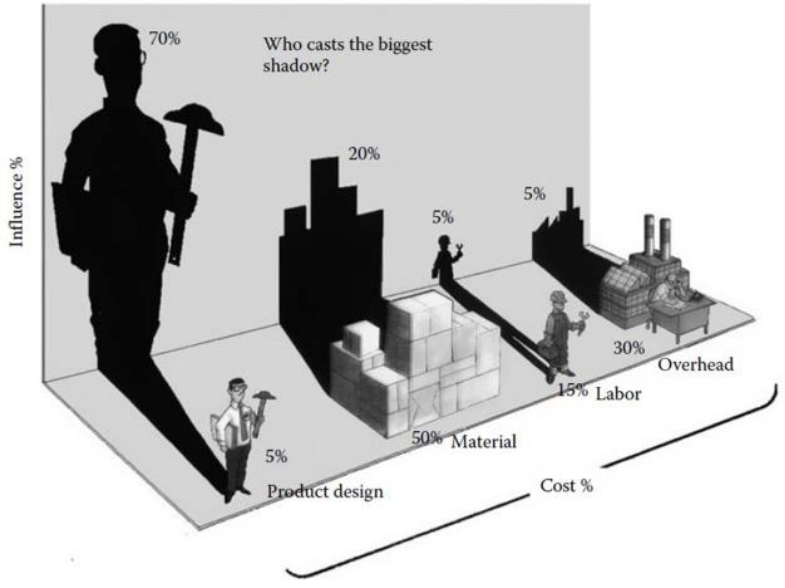

b. "Who casts the biggest shadow?"

Figure 2.3 DFMA impact on production

Figure 2.3 (a), shows that the design procedure with the help of DFMA analysis can lead to a decrease by $40 \%$ of the manufacturing time of a product. In addition to the manufacturing cost of the products, there are other different costs whose reductions contribute to the reduction of final costs. Figure 2.3 (b), is an example of cost reduction, other than manufacturing, after implementing DFMA analysis through an engineering survey [2]. 


\subsection{DFMA working flowchart}

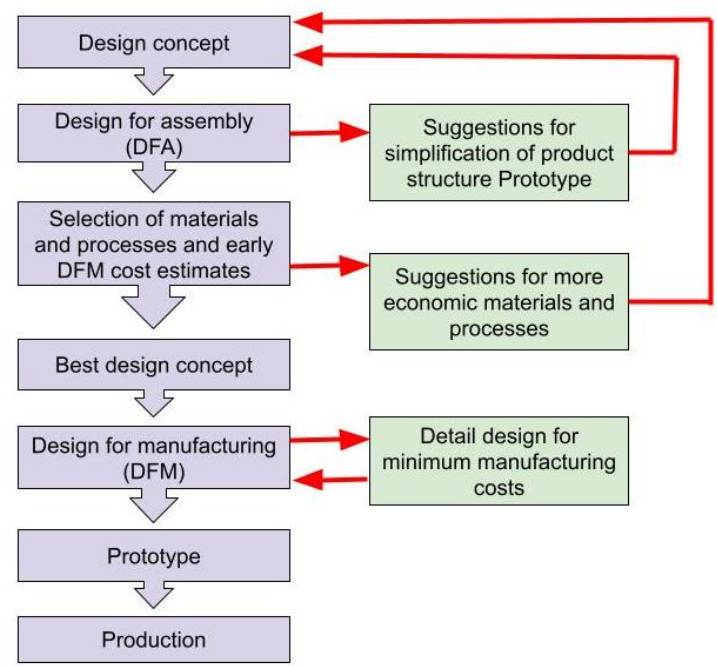

Figure 2.4 Typical steps taken in a DFMA study using DFMA

\subsection{Product analyzed (Tray VLM)}

The product to be analyzed by the DFMA method is a subassembly called Tray VLM, which is part of an automatic storage system called Vertical Lift Module (VLM). This tray has the role of storing various products - with low or relatively high weight - in an orderly manner, respecting certain storage and sorting criteria, defined in the computer of the VLM system [5].

Depending on the construction and design of the tray adopted, it can be more or less modular, so that the user can configure the division of the compartments according to the products to be stored, or for the products that will change during use VLM system, without having to replace the entire VLM Tray.
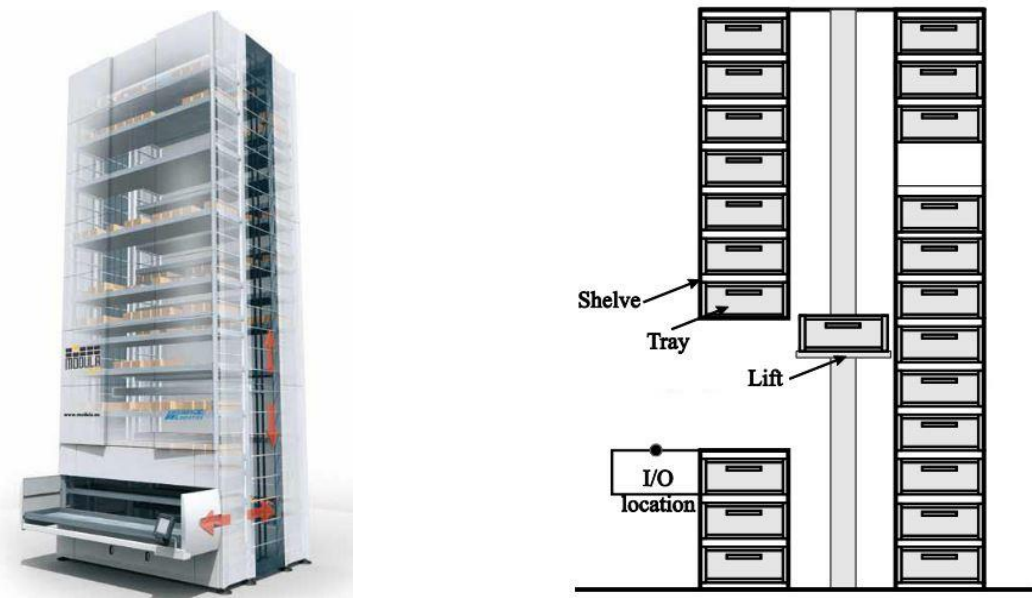

Figure 2.5 Vertical lift module by Modula [8]

Figure 2.5 is represented a diagram with the main elements of such a VLM system, which works based on a computer-controlled elevator, having the role of "collecting" the trays located on the two shelves, arranged in front and behind the elevator, and brought into a position working, input and output (I/O) trays [6].

The purpose of the Tray component analysis was to optimize its modular construction, reduce weight and reduce manufacturing time and cost. 


\section{Analysis of existing design}

\subsection{Understanding the product structure}

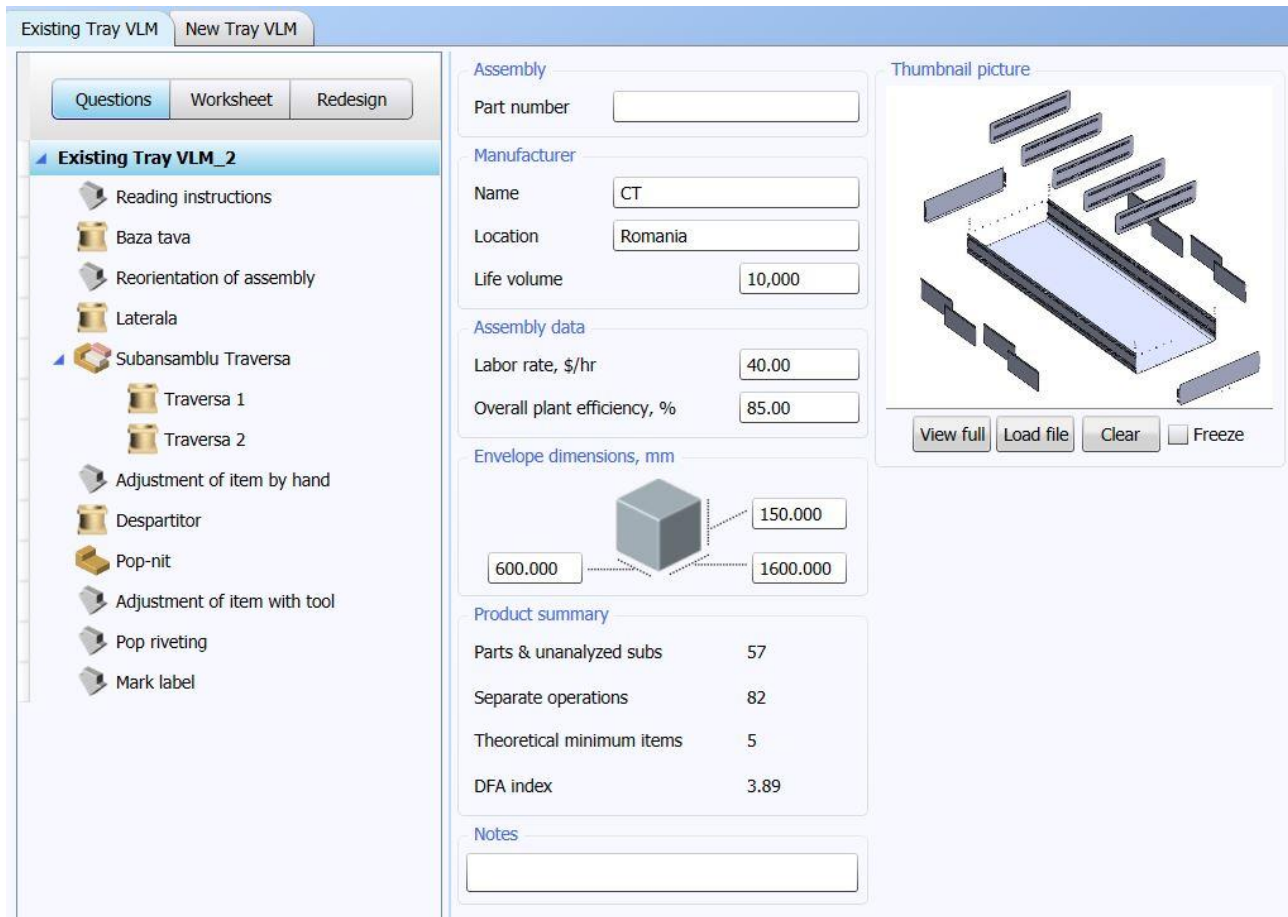

Figure 3.1 Questions for DFA analysis

DFMA analysis begins with some questions related to the product to be analyzed, such as the minimum number of components, the minimum number of fasteners, the use of standardized elements, design to reduce the number of assembly steps and additional operations, and more. Figure 3.1 shows this.

Figure 4.1 is assembled graphically the assembly time for the current product, taking into account the information required in the first stage of analysis.

\subsection{Assembly time and cost estimation}

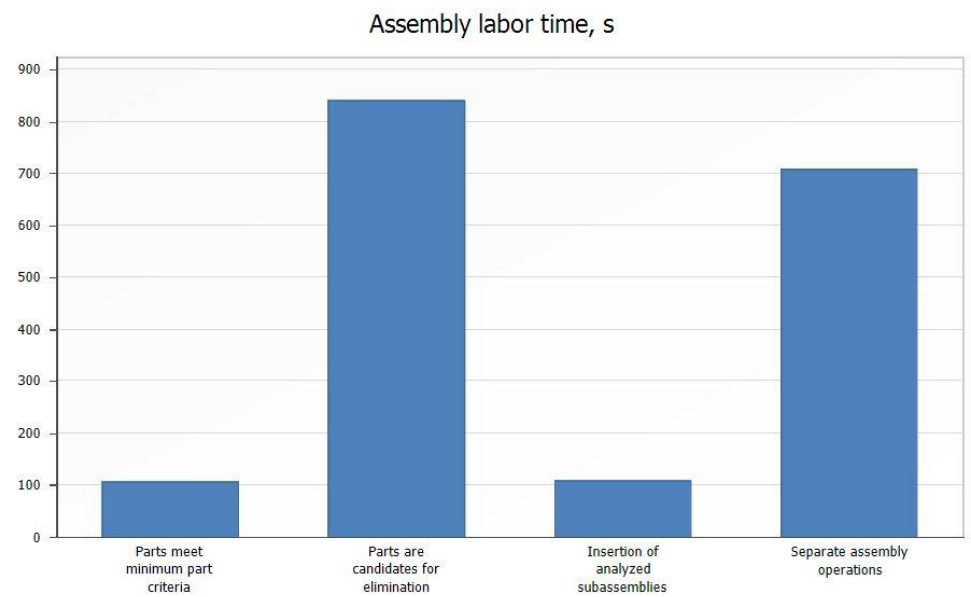

Figure 3.2 Assembly labor time of Existing Tray VLM 
Total assembly process time $=1761.76 \mathrm{~s}$

Total assembly process cost $=23.86 \$$

\subsection{Design efficiency (DFA Index)}

The DFA index is a design efficiency that is calculated by dividing the processing time for an ideal product by the processing time for the actual product [7]. This ratio of ideal process time to actual process time is then expressed as a percentage using the following formula $(0.1)$ :

$$
D F A_{\text {Index }}=\frac{100 \cdot\left[T_{1}+T_{2} \cdot\left(N_{\min }-1\right)\right]}{T_{a}}
$$

Where:

$\mathrm{T}_{1}$ is the ideal assembly process time for handling and inserting the first necessary item in the product;

$\mathrm{T}_{2}$ is the ideal assembly process time for handling and inserting each subsequent necessary item in the product;

$\mathrm{N}_{\min }$ is the theoretical minimum item count;

$\mathrm{T}_{\mathrm{a}}$ is the actual assembly process time for the product.

$\mathrm{T}_{1}$ and $\mathrm{T}_{2}$ differ depending on the size and weight of the product, as well as the $180^{\circ}$ symmetry of the part for at least one of its axes.

All the values mentioned above are calculated by the software based on the justification of the part entries made in the DFA software. The DFA index is calculated in the DFA software at the successful entries.

DFA index for Existing Tray VLM assembly $=3.89$

For improved product design, designers aim to maximize the DFA index.

\section{Redesign changes}

Based on the suggestions of the DFM and DFA software for product redesign, possible design changes were identified to improve the efficiency of the design.

It has been redesigned component „Baza tava”, by adding the two components „Laterala”, to the basic product, this being made from a single product. Thus, reducing the assembly time of the two sides, by removable assembly with pop-rivets. The number of holes related to the assembly with pop-rivets was reduced, also the number of pop-rivets was reduced from 36 pcs to 20 pcs.

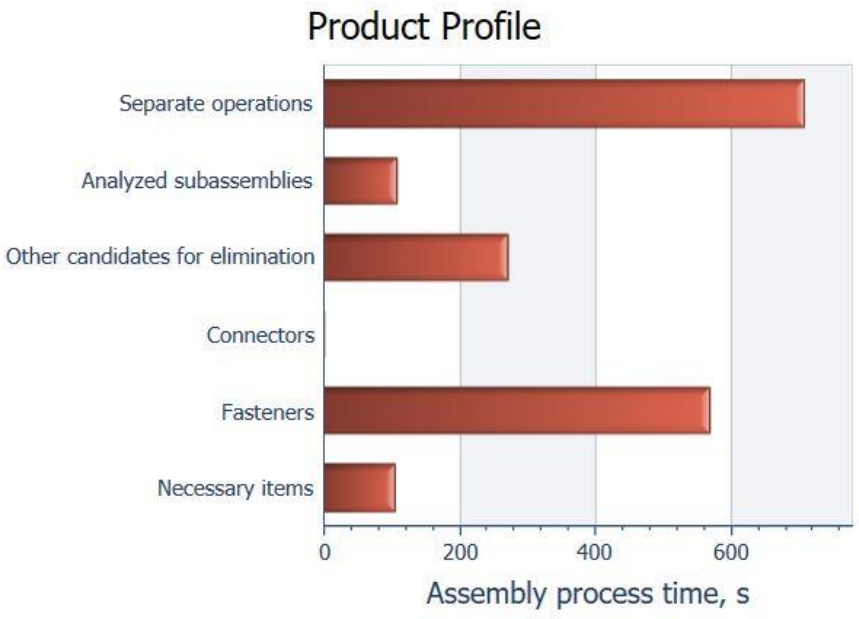

Figure 4.1 Product profile of Existing Tray VLM 


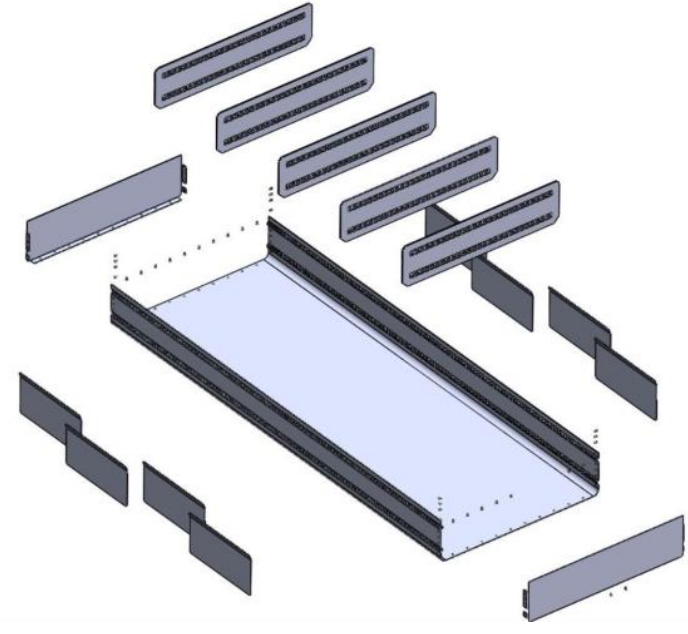

a. After redesign;

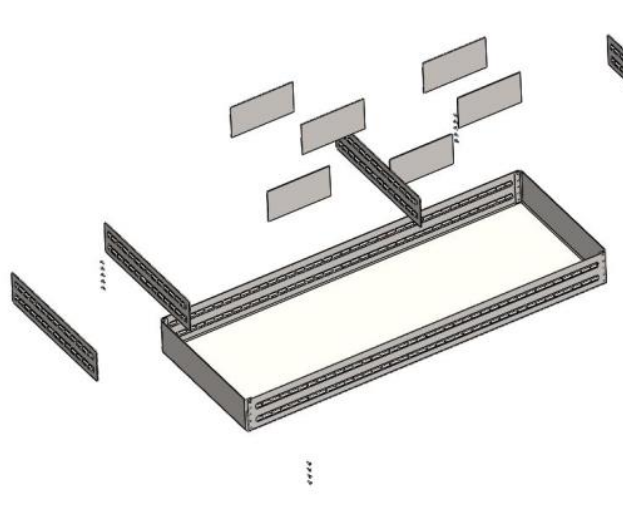

b. Before redesign;

Figure 4.2 After and before redesign Tray VLM

After designing and optimizing the product, in Figure 4.2 the new design obtained is shown, which will be further analyzed with DFMA for the comparison of the results and the validation of its optimization.

\section{Result}

Following the DFMA study, conducted on the two features, DFA and DFM, the results generated by this software can be exported in various forms. The DFA software allows the comparison of results for a maximum of 5 profiles.

Table 5.1 represents the result generated by the system, for the assembly of the Tray VLM product, before and after the DFMA study, analyzing the number of components, the time of the assembly process, and the design efficiency (DFA index).

Figure $\mathbf{5 . 1}$ can be seen graphically, improving the time for the assembly process.

Table 5.1 Tray VLM assembly result

\begin{tabular}{|c|c|c|c|c|}
\hline & $\begin{array}{c}\text { Existing Tray VLM } \\
\text { before DFMA }\end{array}$ & $\begin{array}{c}\text { New Tray VLM } \\
\text { after DFMA }\end{array}$ & Difference & $\%$ \\
\hline \multicolumn{5}{|l|}{ Entries including repeats } \\
\hline Parts meet minimum part criteria & 5 & 4 & -1 & $-20 \%$ \\
\hline Parts are candidates for elimination & 52 & 33 & -19 & $-37 \%$ \\
\hline Analyzed subassemblies & 5 & 5 & 0 & $0 \%$ \\
\hline Separate assembly operations & 82 & 46 & -36 & $-44 \%$ \\
\hline Total entries & 144 & 88 & -56 & $-39 \%$ \\
\hline \multicolumn{5}{|l|}{ Assembly labor time, $\mathrm{s}$} \\
\hline Parts meet minimum part criteria & 105 & 77 & -27.81 & $-26 \%$ \\
\hline Parts are candidates for elimination & 840 & 520 & -320 & $-38 \%$ \\
\hline Insertion of analyzed subassemblies & 109 & 70 & -39 & $-36 \%$ \\
\hline Separate assembly operations & 708 & 426 & -282 & $-40 \%$ \\
\hline Total assembly labor time & 1762 & 1092.56 & -669.2 & $-38 \%$ \\
\hline \multicolumn{5}{|l|}{ Design efficiency } \\
\hline DFA Index & 3.89 & 5.01 & 1 & $29 \%$ \\
\hline
\end{tabular}




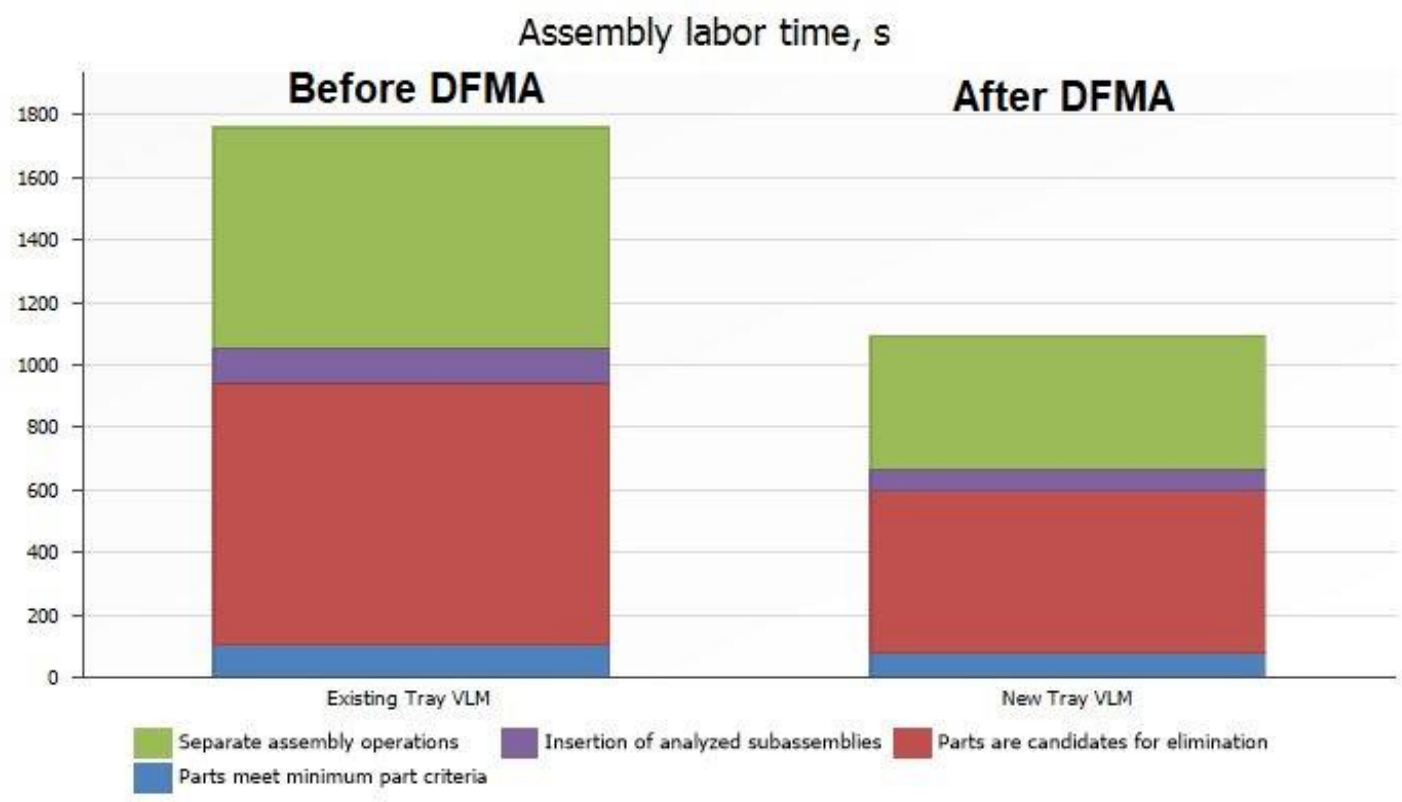

Figure 5.1 Assembly process time of Tray VLM

\section{Conclusion}

The DFMA study was analyzed and successfully applied to the VLM Tray assembly, addressing both the aspects related to the assembly design and the assembly of this product. The basic issues of production and technology have been taken into account since the design stage.

There was a reduction in assembly costs of $37 \%$ and a decrease in assembly process time by $38 \%$. The design efficiency of the assembly was improved by $29 \%$, after a successful analysis with DFMA.

The implementation of the DFMA concept, by using software for such an analysis, in the case of new product development (NPD), can be an added value brought to the newly developed product.

The results of the DFMA have proven to truly support competing engineering.

\section{References}

[1] O. torkelsson Markus Dauksz, "A Methodology Study and Method Development," 2014.

[2] W. A. K. Geoffrey Boothroyd, Boothroyd Dewhurst, Product Design for Manufacturing and Assembly, Third Edit. 2011.

[3] Narcisa VALTER, DESIGN PENTRU FABRICARE SI ASAMBLARE. 2019.

[4] "New Product Development - Enfinio, Inc." http://enfinio.com/new-product-development/ (accessed Oct. 29, 2020).

[5] D. Kolar, G. Dukic, and I. Lucica, "DESIGN OF ORDER-PICKING SYSTEMS WITH VLMs."

[6] B. Rosi, ; Grasic, ; Dukic, ; Opetuk, and T. Lerher, "SIMULATION-BASED PERFORMANCE ANALYSIS OF AUTOMATED SINGLE-TRAY VERTICAL LIFT MODULE,” Int $j$ simul Model, vol. 15, pp. 97-108, 2016, doi: 10.2507/IJSIMM15(1)8.328.

[7] "DFMA ${ }^{\circledR}$ - Cutting Billions in Manufacturing Costs Since 1983 | Boothroyd Dewhurst, Inc." https://www.dfma.com/software/dfma.asp (accessed Apr. 01, 2021).

[8] "Vertical Storage System, Storage Solutions | Modula."

https://www.modula.eu/products/vertical-solutions/ (accessed Apr. 01, 2021). 